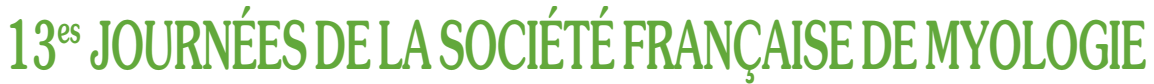 Prix Master 2014

\section{La myostatine}

\section{Une cible thérapeutique potentielle pour les myopathies centronucléaires}

\author{
David Arnould, Anne-Cécile Durieux
}

L'unique modèle murin reproduisant la myopathie centronucléaire autosomique dominante associé à une mutation du gène dynamine-2 (KI-dnm2 $\left.2^{\mathrm{R} 465 \mathrm{~W} /+}\right)$ reproduit la plupart des signes cliniques observés chez l'Homme, notamment une atrophie et une perte de force musculaire. La myostatine (MSTN) est un régulateur négatif majeur du muscle strié squelettique. Nous faisons l'hypothèse que l'inactivation de la mstn pourrait limiter la perte de masse et de force musculaire chez la souris KI. Afin de valider cette hypothèse, nous avons généré une souris doublement mutée (KIKO) par croisement entre souris KI et souris inactivée pour la mstn (KO-mstn). Les animaux sont suivis durant 12 mois. La force musculaire et la motricité ont significativement été altérées chez la souris KI à 1 mois. Une perte significative de masse et du volume musculaire (microIRM) a été observée à partir de 2 mois. Entre 2 et 12 mois, tous ces paramètres restent en dessous des valeurs contrôles. En comparaison de la souris $\mathrm{KI}$, la souris KIKO présente une augmentation de la force musculaire ainsi qu'une capacité motrice moins affectée. De plus, la masse et le volume musculaire ont été augmentés dès l'âge de 1 mois. Les analyses moléculaires montrent que l'inactivation de la mstn entraîne une augmentation du niveau d'expression de différentes protéines impliquées dans la voie IGF1/Akt/mTOR, mais aussi une diminution du niveau d'expression de différents acteurs de la voie de dégradation ubiquitine-protéasome. L'inactivation de la mstn montre une amélioration de la masse et de la fonction musculaire chez la souris KI. À l'avenir, nous souhaitons intervenir après la mise en place de la pathologie, par injection d'une drogue anti-mstn permettant de piéger la molécule. Mots clés : myopathie centronucléaire ; dynamine ; myostatine ; modèles animaux.

La myopathie centronucléaire autosomique dominante (MCN-AD) est une maladie musculaire congénitale rare liée à des mutations majoritairement retrouvées dans le gène dynamine 2 (DNM2) [1]. Les caractéristiques cliniques reportées sont notamment une atrophie et une faiblesse musculaire progressives. La MCN-AD est peu sévère, lentement progressive et représente $50 \%$ des $\mathrm{MCN}$. Les patients atteints de MCN-AD développent une faiblesse musculaire localisée préférentiellement au niveau des muscles du visage, des muscles proximaux, et le plus souvent associée à un ptosis ou une ophtalmoplégie [2]. L'ensemble des caractéristiques cliniques observées conduit à terme à une détérioration de la qualité de vie des patients. Bien qu'aucune thérapie curative ne soit actuellement disponible, de nombreux efforts sont réalisés afin de proposer une prise en charge du déficit musculaire associé. La taille du muscle dépend d'un équilibre dynamique entre la dégradation et la synthèse de ses protéines constituantes [3]. Les voies autophagie-lysosome et ubiquitine-protéasome (UP) sont les voies principales de dégradation des protéines musculaires. La voie de signalisation IGF1/Akt/mTOR représente la voie principale de stimulation de la synthèse des protéines dans le muscle. Toute action sur cette balance dynamique peut conduire à l'hypertrophie et inversement à l'atrophie du tissu musculaire. La connaissance des mécanismes cellulaires et moléculaires de régulation de la masse musculaire en conditions normales, ainsi que la nature de leur dysfonctionnement dans la physiopathologie de la MCN-AD, permettrait d'imaginer des interventions sur ces voies de contrôle de la masse musculaire, en conditions pathologiques. C'est le cas par exemple, pour la maladie de Pompe, caractérisée par une surcharge lysosomale due au déficit d'une enzyme. Le traitement enzymatique substitutif de la société Genzyme Myozyme $^{\circledR}$ alpha alglucosidase agit directement sur la voie de dégradation autophagie-lysosome en 
permettant la dégradation des surcharges lysosomales inhérentes à la pathologie.

La myostatine (mstn) est un facteur de croissance spécifique du muscle strié squelettique qui lorsque son expression/signalisation est bloquée conduit à l'acquisition d'un phénotype hypermusclé [4].

Dans le modèle murin $m d x$, modèle pour la myopathie de Duchenne, il a été reporté une augmentation de la taille des fibres, associée à une augmentation de la masse et de la force musculaire lorsque le gène de la mstn était inactivé [5]. Une telle stratégie mstn interférente a également été utilisée sur un modèle de myopathie myotubulaire ( $\mathrm{MCN}$ liée à une mutation de MTM1) [6]. Dans ce cas, il a été montré une amélioration du phénotype musculaire mais seulement de façon transitoire. En effet, cela ne permettait pas l'allongement de la durée de vie qui dans ce modèle animal sévère est de l'ordre de 8 à 9 semaines. Récemment, une invalidation de la mstn chez des souris développant une cachexie liée au cancer du côlon a prolongé leur durée de vie de façon spectaculaire (doublement de la durée de survie) associée à une diminution de l'évolution du nombre de polypes intestinaux [7]. L'inactivation de la mstn pourrait être un outil thérapeutique envisageable dans des pathologies musculaires de faible sévérité reportée chez l'animal modèle. Le but du projet primé par le prix Master 2014 de la Société Française de Myologie consiste à évaluer la faisabilité et l'efficacité d'une stratégie mstn interférente dans le seul modèle murin actuellement disponible pour la MCN-AD. Ce modèle porte la mutation la plus fréquemment retrouvée dans les cas de $\mathrm{MCN}-\mathrm{AD}$, localisée dans le gène de la dynamine 2 (dnm2). Le modèle Knock-In KI-Dnm-2 ${ }^{\mathrm{R} 465 \mathrm{~W} /+}$ (KI) est une souris porteuse à l'état hétérozygote d'une mutation faux sens donnant lieu au remplacement d'un acide aminé arginine $\mathrm{R}$ en position 465 par un acide aminé tryptophane $\mathrm{W}$. Les souris $\mathrm{KI}$ exprimeront à la fois une version normale et mutée du gène $d n m 2$. Bien que le modèle soit déjà décrit $[8,9]$, les conséquences fonctionnelles de la mutation sur la fonction de la protéine restent mal connues.

Nous avons émis l'hypothèse selon laquelle l'inactivation du gène de la mstn pourrait être bénéfique à la souris KI, en limitant/stoppant la perte de masse et de force musculaire. Pour répondre à cette question, les souris $\mathrm{KI}$ ont été croisées avec des souris $\mathrm{KO}-m s t n(\mathrm{KO})$, porteuses à l'état homozygote d'une mutation aboutissant à l'excision de l'exon 3 du gène de la mstn, conduisant à la synthèse d'une protéine tronquée non fonctionnelle entraînant un phénotype hypermusclé (modèle KIKO).

Nous avons étudié l'évolution du poids et le volume des différents muscles postérieurs durant 12 mois.
Parmi ces différents muscles, le Tibial antérieur présente chez la souris $\mathrm{KI}$, une atrophie lentement progressive correspondant à l'évolution connue de la maladie chez l'homme. En effet, à 1 mois nous n'observons pas de diminution du poids et du volume musculaire chez la souris KI. Nous observons cependant une diminution de ces deux paramètres dès l'âge de 2 mois et toujours présente à 8 et 12 mois. L'ensemble des analyses biochimiques et histologiques sera réalisé sur ce muscle.

Les premiers résultats des effets de l'inactivation de la mstn sont encourageants puisqu'ils démontrent un bénéfice certain dans ce contexte pathologique avec une augmentation du poids du muscle Tibial antérieur. L'étude microIRM réalisée au CERMEP de Lyon met également en évidence une augmentation du volume du muscle Tibial antérieur. De façon concomitante, les animaux présentent une amélioration de la force musculaire et de leur capacité locomotrice.

Au niveau moléculaire, l'analyse des voies de régulation de la masse musculaire montre que l'inactivation de mstn permet de diminuer le niveau d'expression de certains acteurs impliqués dans la voie de dégradation ubiquitine-protéasome et d'augmenter celui d'acteurs impliqués dans la voie stimulant la synthèse des protéines, la voie IGF1/Akt/mTOR. Ces résultats nécessitent d'être approfondis afin d'affiner nos conclusions sur les mécanismes moléculaires impliqués, mais aussi de s'assurer de l'absence d'effet délétère d'une telle stratégie.

Une étude histologique (coupe transversale du muscle Tibial antérieur) est en cours pour compléter l'ensemble des résultats de mes $1^{\text {re }}$ et $2^{e}$ années de thèse. Nous utiliserons la coloration Hématoxyline Éosine (HE), afin de visualiser l'état général du muscle. La coloration Nicotinamide adenine dinucleotide reduced (NADH) - tétrazolium réductase (TR), reflet du nombre de mitochondries, permetégalement la mise en évidence du réticulum sarcoplasmique. Elle nous permettra par ailleurs de mettre en évidence des anomalies de structures, observées à partir de deux mois chez la souris et proche de ce qui est également caractéristique de la MCN-AD chez l'homme. De plus, la coloration NADH, nous informera sur le typage des fibres musculaires. Des colorations immunohistochimiques permettant de mettre en évidence des protéines impliquées dans les voies de signalisation précédemment étudiées en $q P C R$ et Western blot, seront également réalisées.

Compte tenu des résultats encourageants que nous avons obtenus, nous allons évaluer l'efficacité d'un traitement chez l'animal visant à piéger la mstn circulante. Ceci est un préambule indispensable afin d'envisager à plus long terme la transposition d'une telle application en situation clinique. 


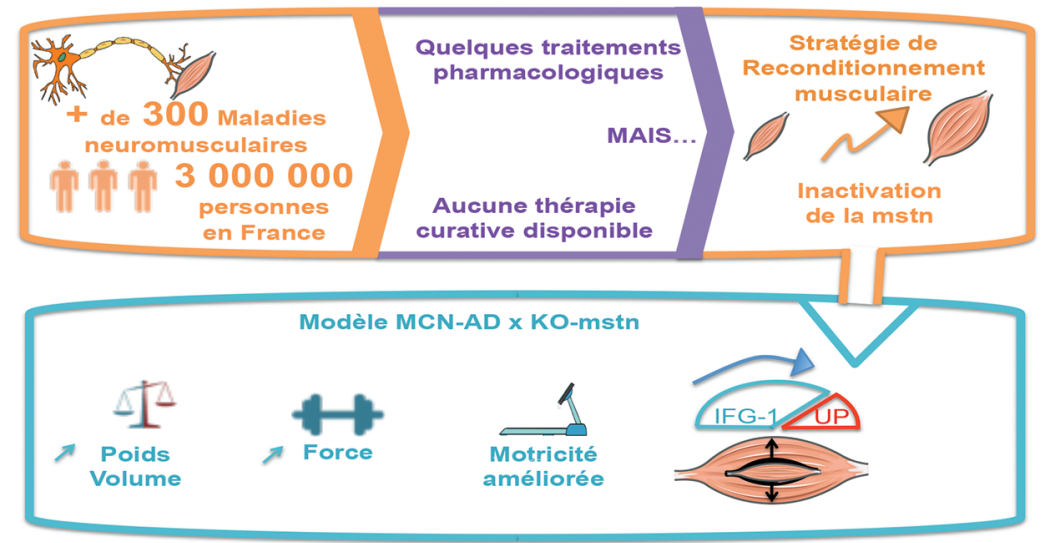

Figure 1

MNM et bénéfice d'une stratégie mstn interférente. Avec environ 3 millions de personnes atteintes de MNM en France et aucune thérapie curative disponible, il y a nécessité de développer des stratégies de reconditionnement musculaire. L'inactivation de la mstn est une piste thérapeutique intéressante. Dans le cas de la MCN-AD, nous avons démontré le bénéfice évident sur la physiologie musculaire d'une telle stratégie : augmentation du poids et du volume musculaires associée à une amélioration la force musculaire et la capacité locomotrice. Au niveau moléculaire, l'inactivation de la mstn permet une stimulation de la voie de signalisation enclenchée par l'IGF-1, et une atténuation de la voie de dégradation ubiquitine-protéasome (UP). Ces deux événements contribuent à l'amélioration du phénotype musculaire lié à la maladie.

\section{Abstract}

Master Award 2014

Myostatin: a potential therapeutic target in centronuclear myopathies

The unique mouse model for autosomal dominant centronuclear myopathy (KI-dnm $\left.2^{R 465 \mathrm{~W} /+}\right)$, associated to mutation of the dynamin-2 gene (dnm2) reproduces some of the human clinical features, notably muscle atrophy and weakness. Myostatin (MSTN), is a master negative regulator of skeletal muscle mass. We hypothesized that inactivation of mstn could limit muscle atrophy and weakness reported in the KI mouse. To validate this, we intercrossed KI mice with mice inactivated for mstn (KO-mstn) to generate a double mutated lineage (KIKO mice). Animals were followed during 12 months. Muscle force, and locomotor activity were significantly altered in 1 month-old KI mice. A significant loss of muscle mass and volume (microRMI) was observed in KI mice from 2 months of age. From 2 to 12 months, all these parameters remained below of control values. When compared to KI mice, KIKO mice presented an increase of muscle grip strength and less affected motor skills. In agreement with these data, muscle mass and volume were increased at all ages. Molecular analyzes showed that inactivation of mstn allowed for an increase of several proteins involved in the IGF1/Akt/mTOR pathway, but also a down regulation of several factors involved in the ubiquitin-proteasome pathway. Overall, we demonstrated that inactivation of mstn improves muscle mass and function of KI mice. These results are very promising since genetic inactivation of mstn shows a real benefit for KI mice. The perspective to this work is to evaluate the efficiency of an anti-mstn based pharmacological approach to restore muscle function after the establishment of the disease.

Key words: centronuclear myopathy - dynamin myostatin - animals models

\section{LIENS D'INTÉRÊT}

Les auteurs déclarent n'avoir aucun lien d'intérêt concernant les données publiées dans cet article.

\section{RÉFÉRENCES}

1. Bitoun M, Maugenre S, Jeannet PY, et al. Mutations in dynamin 2 cause dominant centronuclear myopathy. Nat Genet $2005 ; 37$ :1207-9.

2. Romero NB. Centronuclear myopathies: a widening concept. Neuromuscul Disord 2010 ; 20 : 223-8.

3. Freyssenet D. À propos des mécanismes de contrôle de la masse musculaire. Cahiers de Myologie octobre 2009; $\mathrm{n}^{\circ} 1$ : 11-3.

4. McPherron AC, Lawler AM, Lee SJ. Regulation of skeletal muscle mass in mice by a new TGF-beta superfamily member. Nature 1997 ; 387 : 83-90.

5. Amthor H, Hoogaars WMH. Interference with myostatin/ ActRIIB signaling as a therapeutic strategy for Duchenne muscular dystrophy. Curr Gene Ther 2012; 12 : 245-59.

6. Lawlor MW, Read BP, Edelstein R, et al. Inhibition of activin receptor type IIB increases strength and lifespan in myotubularindeficient mice. Am J Pathol 2011 ; 178 : 784-93.

7. Gallot YS, Durieux AC, Castells J, et al. Myostatin gene inactivation prevents skeletal muscle wasting in cancer. Cancer Res $2014 ; 74$ : 7344-56

8. Durieux AC, Vassilopoulos S, Lainé J, et al. A centronuclear myopathy: dynamin 2 mutation impairs autophagy in mice. Traffic $2012 ; 13$ : 869-79.

9. Durieux AC, Vignaud A, Prudhon B, et al. A centronuclear myopathy-dynamin 2 mutation impairs skeletal muscle structure and function in mice. Hum Mol Genet 2010; 19: 4820-36.

\section{Abréviations}

Akt : protéine kinase B ; dnm2 : dynamine 2 ; IGF-1 : insulin-like growth factor 1 ; Mstn : myostatine ; mTOR : mammalian target of rapamycin. 